\title{
Consumers' Sentiments and Popularity of Brand Posts in Social Media: The Moderating Role of Up-votes
}

\author{
Hamid Khobzi, Raymond Y.K. Lau, Terence C.H. Cheung \\ Department of Information Systems, \\ City University of Hong Kong, \\ Hong Kong, China \\ hkhobzi2-c@my.cityu.edu.hk, \{raylau, is.tc \}@ cityu.edu.hk
}

\begin{abstract}
User-generated contents (UGC) on online social media plays an important role in the branding and marketing of firms' products and services. In this study, we examine the effect of consumers' sentiments embedded in UGC on the popularity of brand posts. We retrieved real-world data from a social media platform and utilized a rigorous data analysis method that exploited state-of-the-art semi-supervised sentiment analysis technique. Our empirical findings confirm that positive and negative sentiments are associated with post popularity to some extent. Also, the customers' up-votes for negative comments somehow moderates the effect of negative comments on post popularity. To the best of our knowledge, this is the first study that demonstrates the specific role of upvotes in enhancing the popularity of brand posts on online social media. Our findings provide a promising theoretical contribution to the literature. The managerial implication is that firms can apply our findings to develop more effective strategies for marketing through social media brand communities.
\end{abstract}

Keywords: Social Media, Online Brand Community, User-generated Contents, Sentiment Analysis.

\section{Introduction}

By pervasiveness of social media in past decade, a new dimension of interactions between customers and firms is formed. Social media are a proper means of interaction between firms and customers from which both sides can benefit a lot. In this regard, social media brand communities that are a special form of online brand community, can help firms to achieve higher levels of loyalty from their customers and improve the relationship with their customers [1], [2]. Meanwhile, realizing consumers' online activities is crucial for firms because consumers can easily involve in discussions and express their opinion about the firm to the others [3]. In social media brand communities, also referred as brand fan pages on social media (see [4], [5]), consumers are also able to click on the "like" button or submit comments in relation to every brandrelated post. Also, they may be able to react to others' comments by liking (or up-voting) their comments. On the other hand, firms desire to obtain the biggest possible dissemination for their brand-related posts among social media users. By brand-related posts, we mean any sort of content that is posted on the online brand community by its administrators but not regular customers. It can be a product advertisement, promotion, or a news about the firm. But consumers are very important elements in an efficient dissemination for brand-related posts among the users of social media. More consumers engage with a post, possibly more views and attention the post gains by other users. Therefore, brand-related posts gain more popularity by this mechanism. This is undoubtedly one of the important aims social media marketers in particular.

There are various studies illustrating the advantages that social media provide for firms. For example, firms can improve their financial performance in long-term by using social media [6]. Also, customers' purchase behavior is strongly affected by UGC in the brand community of company [7]. UGC embedded in social media can be used for developing potential innovations in firm [8], or to estimate company's online reputation [9]. Especially, social media have offered comprehensive information regarding Customer-toCostumer (C2C) interactions such as word-of-mouth (WOM) communications that was not available formerly [10].

We can consider consumers' comments as the most informative and important type of consumers' online activities. A consumer can express his opinion about a brand-related post and it will be exposed to other consumers. Similar to product reviews written by customers of an online shop, comments on a social 
media brand community can be considered as the electronic word of mouth (eWOM) and they carry different sentiments regarding a post [11]. Sentiment analysis as an opinion mining approach is an emerging approach in the context of social media. It is a useful approach for processing social media textual content to explore customers' opinions about marketing campaigns or product preferences [12]. However, there are relatively few studies that examined consumers' sentiments and their outcomes for business in social media brand communities context.

By considering aforementioned facts about the importance of the context, we tried to focus on online $\mathrm{C} 2 \mathrm{C}$ interactions in social media and explore consumers' sentiments that are embedded in postrelated comments in different social media brand communities. To address the existing gap in the literature and importance of the topic, our study seeks to find a proper answer for the following proposed research question:

RQ1) How and to what extent is the popularity of posts affected by different consumers' sentiments in social media brand communities?

\section{$R Q 2)$ What is the role of consumers' up-votes in gaining popularity for brand-related posts?}

In this regard, we tried to examine the relationship between consumers' sentiments embedded in their comments on post popularity and see if such a relationship can be moderated by consumers' up-votes in the comment section. Our empirical study presents interesting insights about factors associated with the popularity of posts in brand communities of social media using real-world data retrieved from Facebook. For instance, when consumers express more negative sentiments in their comments in relation to a post it can be unfavorable for the popularity of the post. Meanwhile, if more negative comments garner more up-votes, it positively amplifies the shares' count and conversely inhibits likes' count and comments' count of the post. This research is conducted utilizing a rigorous data analysis to provide new insights about consumers' sentiments and post popularity and presents a useful theoretical contribution. This approach will be helpful to provide business intelligence for firms so as to improve their social media marketing strategies.

\section{Research background}

The virtual or online brand communities have been concentrated by scholars and practitioners of
Information Systems and Marketing over the past decade. In this regard, the social media brand community is a more recent area of focus for these scholars and practitioners. Although, social media brand communities are a sort of virtual brand community, there are several important differences between them such as social context, scale, and content (see [1]). Thus, we exclusively focus on social media brand communities in this study.

The majority of prior studies in the context of social media brand communities had a greater focus on Marketer-generated Content (MGC) features rather than UGC features (e.g. [13]-[15]). In a primary research, De Vries et al. [13] focused on identifying the factors that can affect post popularity in social media brand communities. They provided useful insights by analyzing brand-related posts from various international brands; For instance, if a post contains positive comments more than negative ones, there would be a positive impact on post likes' count. Sabate et al. [14], analyzed posts from several different travel agencies and they showed content richness is an important influencing factor to gain more likes' count for a post. On one hand, putting website links in the content of a post may have a negative impact on comments' count. On the other hand, providing images in the content of a post and a choosing an appropriate time to publish the post on the brand community would have a significant effect on comments' count. Zadeh and Sharda [15] proposed a model to predict the popularity of posts in social media brand communities considering two factors of time and followers' count.

There are other studies that investigated the plausible impact of MGC and UGC on the growth of social media brand community (i.e. the rate of increase of community members) [16] or on the consumers' awareness, interest, and purchase decision [17]. Similarly, Xie and Lee [4], first referred to UGC and MGC in social media brand communities as earned and owned social media activities, respectively and then examined the impact of these activities on purchase decision making of consumers by presenting a twostage model. Wang et al. [18], investigated the effect of Firm-generated Content (i.e. MGC) and UGC of Facebook fan pages on offline purchase behavior of consumers in the U.S. automobile industry. According to the aforementioned studies, UGC is less considered for studying factors influencing post popularity in social media brand communities.

In the context of social media brand communities, the number of research studies using sentiment analysis is relatively small. For example, Goh et al. [7] tried to extract information and persuasion features of UGC and MGC using a text mining tool that employs a lexicon for sentiment analysis. They examined how 
those features influence purchase behavior of consumers by considering directed and undirected communications between marketers and consumers. Finally, they showed that UGC has a stronger influence on consumer purchase behavior rather than MGC. Khobzi and Teimourpour [5] examined the significance of the relationship between comments' polarity and popularity of posts using a lexicon-based approach for sentiment analysis. They determined polarity as a sentiment feature equal to the count of positive lexicon terms minus the count of negative lexicon terms in a comment. This definition is similar to the way Lin and Goh [19] defined a UGC feature called information favorableness. They examined the impact of information favorableness and information richness of UGC and MGC on firm's sales performance by using same sentiment analysis approach as Goh et al. [7]. Similarly, Tang et al. [20] tried to apply an analogous sentiment analysis approach on UGC retrieved from Facebook and YouTube and specifically focused on neutral UGC and examined its impact on product sales. Wu et al. [21], tried to analyze consumers' emotional biases in an online brand community by utilizing sentiment analysis in order to classify them into two distinguished groups; Then, they analyzed the influence of level of activity of each group in the brand community on their purchase frequency.

According to the reviewed literature, there are a few gaps in the literature that we tried to address in this research. First of all, we have focused on the unique role of neutral sentiment in the popularity of posts in social media brand communities along with positive and negative sentiments. Secondly, we considered shares' count as a proxy of post popularity. Finally and most importantly, we examined the specific role of consumers' up-votes as the moderator of the relationship between consumers' sentiments and post popularity.

\section{Research methodology}

\subsection{Theoretical foundation and hypotheses}

Based on Information Asymmetry, two different parties (sellers and buyers) can access to varying levels of information. For instance, we can clearly observe Information Asymmetry in the current status of online marketing [22]. When the seller provides product price with no more information on an online shopping website, Information Asymmetry exists in such a circumstance. In e-business, buyers usually confront Information Asymmetry because it is not possible to physically evaluate product quality or easily judge the trustworthiness of seller [23]. In such circumstances, website signals are a good source of information to help consumers judge the quality of product or seller. This information seeking tendency of individuals exists in social media as well. Drawing on Information Asymmetry, Goh et al. [7], declared that customers tend to obtain required information from marketers and other customers in social media brand communities while sellers' related signals are supposed to be more effective in comparison to buyers' related signals. However, their results illustrated that both information richness and valence of UGC outperform those from MGC in terms of a significant influence on purchase behavior of consumers.

When consumers participate in activities related to a post such as liking, the post achieves more views among users of social media. For example, when a consumer shares a post on his profile, write a comment on the post, or like the post by clicking on "like" button on Facebook, a percentage of his friends can see the corresponding activity log in their home page. Hence, that post appears on other users' home page and they can also involve with it. Therefore, the corresponding post certainly achieves more views. This process indicates the process of information dissemination in social media. This process can help firms to crowdsource their marketing in social media [15] that exists in other social media platforms such as Instagram as well. Accordingly, we can assume likes' count, comments' count and shares' count of a post represent the extent of dissemination among users of social media or in other words post popularity. Although Likes' count and comments' count is considered as post popularity related proxy variables in prior research [5], [13], [14], shares' count is gained less attention as a proxy for post popularity in prior studies [24]. We considered all three variables as good proxies of post popularity in social media brand communities; while each one of them portraits different level of engagement and post popularity consequently. For instance, sharing a post seems to better disseminate the post and garner more post popularity as a result comparing to liking a post. But these differences are unknown.

Based on what we mentioned above, consumers tend to engage in post-related activities and consume contents such as reading post-related comments written by other consumers. Although some consumers may not read any comment, some of them tend to read one or more comments. Participation of this portion of consumers in post-related activities may be influenced by the exposure to different sentiments that other consumers expressed in comments. Therefore, we assume consumers' expressed sentiments in postrelated comments might have an effect on the 
popularity of posts in social media brand communities. De Vries et al. [13], showed that sharing positive comments on posts is positively related to post popularity. Also, posting positive comments positively influences the purchase intentions of individuals [25]. Based on a general consensus, people's attitude toward products and purchasing them can be increased or decreased by exposure to positive and negative UGC respectively [20]. For instance, positive WOM on twitter is positively associated with movie sales and negative WOM on twitter is negatively associated with movie sales [26]. Accordingly, we propose following hypotheses:

H1) Exposure to positive comments is positively associated with post popularity.

H2) Exposure to negative comments is negatively associated with post popularity.

In the majority of studies that are already mentioned, authors overlooked the neutral sentiments in post-related comments. They focused on subtraction of positive and negative sentiments in post-related comments [7] or post popularity [5]. Tang et al. [20], tried to examine the influence of neutral comments on product sales; In this regard, they classified neutral comments into two groups of mixed-neutral (i.e. equal combination of positive and negative sentiments) and indifferent-neutral (i.e. purely neutral) and showed they have a positive and negative impact on product sales respectively. In our study, these two groups fall into a single group as neutral comments. Sonnier et al. [27], showed a positive relationship between neutral comments and sales performance. Also, neutral UGC has a positive effect on the rate of those customers who are persuaded in a firm's Facebook page to visit and buy products from its online shop [17]. Accordingly, we propose the hypothesis $\mathrm{H} 3$ in the following:

\section{H3) Exposure to neutral comments is positively associated with post popularity.}

In some social media platforms such as Facebook, not only can users engage with the posts, but also other users' comments. One of the ways to engage with a user's comment is to click on the "like" button associated with the comment, or in other words, to "upvote" the comment. There may be a handful of studies that investigated the up-voting behavior in an online environment to provide a better understanding of the antecedents that affect such behavior (e.g. [28]-[30]). However, our study focuses on the consequences of up-voting the users' comments. When more users upvote others' comments, it will have different consequences. For instance, when a comment gains a lot of up-votes by other users, it shows that all those users might have the same opinion [31] or they support the expressed opinion [32]. It may affect the perception of consumers about the reliability of the comments.

By default, those comments with higher up-votes will be promoted and positioned firstly in the list of comments and will be more prominent [29], [33]. On the contrary, comments with less or no up-votes will be positioned at the lower places in the list of comments and users need to scroll down to observe them. Majchrzak et al. [34], referred to up-voting a comment as a sort of "metavoicing" and declared that metavoicing is likely to either foster or inhibit the productivity of the knowledge conversations in different circumstances. Similarly, up-voting may increase the informational exchange on a Q\&A website [35]. Accordingly, we assume that based on the number of up-votes that consumers' comments gain, the impact that the consumers' sentiments have on proxy variables of post popularity will be moderated. Thereby, we propose the following hypotheses:

H4a) The effect of exposure to positive comments on post popularity is stronger in the presence of higher up-votes.

H4b) The effect of exposure to negative comments on post popularity is stronger in the presence of higher up-votes.

\section{H4c) The effect of exposure to neutral comments on post popularity is stronger in the presence of higher up-votes.}

\subsection{Data description}

We retrieved data from Facebook pages of famous international brands. A Facebook page (or fan page) is considered as a social media brand community that every firm (or brand) can establish one and interact with its consumers. We found the official Facebook pages of most valuable brands in Technology sector according to the Forbes ${ }^{1}$ ranking (accessed on November 23, 2015). 13 out of 15 brands in this Technology sector had an official Facebook Page. Thus, we focused on those 13 brand communities to collect required data. We connected to Facebook API using $\mathrm{R}$ programming language to retrieve data from those 13 brand communities. We collected data related to last 50 posts of each brand community to prepare a

\footnotetext{
${ }^{1}$ http://www.forbes.com/powerful-brands/list/
} 
balanced panel data. We repeated data retrieval for same posts for 7 calendar days (i.e. one week). We collected data related to 650 posts from the whole brand communities every day. To serve this purpose, we developed a program by $\mathrm{R}$ programming language which retrieved posts-related data, cleaned them and stored the required data in a research database. Finally, we used the data which was stored in the database for further data processing.

Afterward, we mined content of comments using acknowledged and reliable sentiment analysis approaches by implementing them in $\mathrm{R}$ programming language along with Python. It should be mentioned that we did not desire to propose a new sentiment analysis algorithm in this study. Due to the number of comments that need to be analyzed, which was relatively large (more than 380,000 cumulatively), we could not apply only a supervised classification approach for sentiment analysis. Supervised classification needs a relatively big training sample including labeled comments. However, all retrieved comments are unlabeled. Therefore, we applied semisupervised classification because it needs a small training sample. To provide this small training sample, we used lexicon-based sentiment analysis to label a small percentage of comments $(5 \%)$. Then, we trained semi-supervised sentiment analysis algorithm with provided training sample. Before we start sentiment analysis process, we cleaned all comments to keep the pure text. So, any comment consisting of anything except text such as emoticons or hyperlinks were removed from the analysis. Also, we only kept comments with English language and removed all nonEnglish comments from our analysis. We considered three categories of positive, negative, and neutral for classification of consumers' comments.

In order to provide a small labeled sample of comments, we employed Naïve Bayes classification method along with the subjectivity lexicon proposed by Wiebe and Mihalcea [36]. This method computes the probability of membership in each sentiment class for each comment based on the commonality of terms in the comment and the lexicon. Then, it assigns the sentiment class with the superior likelihood to the comment. Afterward, we applied Label Propagation as a reliable graph-based method [37] on whole comments to classify them by using the labeled output of the previous step as the training sample. The general idea of Label Propagation is that it builds a graph in a way that all data points including both labeled and unlabeled points are its nodes and similarities between data points are represented by edges. It uses labeled nodes as a source of information and propagates the information through the whole graph in order to assign a label to every node in the graph. We identified the frequency of each expressed sentiment for every post and stored the results in a new dataset for all posts. This measurement for different sentiments is similar to what have been used prior research (see [20]). Finally, we stored the values of all variables for each post and each day in a new dataset to use for testing the proposed hypotheses.

A newly published post has more chance to be disseminated among consumers rather than old posts. In other words, older posts may be less impactful than the new ones [6]. Because new posts appear on the homepage of the followers of the brand community. However, consumers should visit the brand community page and scroll down to see old posts and engage with them. That is why the time interval between the time a post is created and the time of our data collection is important to notice. Therefore, we controlled for post creation interval (PCI) as a time lag for brand-related posts. We carried out all these intensive procedures only to prepare a reliable dataset for testing the proposed hypotheses. Table 1 shows the descriptive statistics of our final dataset.

Table 1. Descriptive statistics of the final dataset

\begin{tabular}{|c|c|c|c|c|c|}
\hline Variable & Mean & $\begin{array}{c}\text { SE. } \\
\text { Mean }\end{array}$ & Min & Max & Std. Dev \\
\hline$L$ & $\begin{array}{c}20246.2 \\
1\end{array}$ & $\begin{array}{c}1378.75 \\
8\end{array}$ & 1 & 904513 & $\begin{array}{c}92715.7 \\
3\end{array}$ \\
\hline$C$ & 725.084 & 47.27 & 0 & 44918 & 3178.72 \\
\hline$S$ & $\begin{array}{c}2530.60 \\
4\end{array}$ & 430.52 & 0 & 696321 & $\begin{array}{c}28950.6 \\
5\end{array}$ \\
\hline$P O S$ & 77.197 & 2.125 & 0 & 989 & 142.907 \\
\hline$N E G$ & 5.519 & 0.161 & 0 & 124 & 10.856 \\
\hline$N E U$ & 2.993 & 0.11 & 0 & 118 & 7.375 \\
\hline$U V R_{(P O S)}$ & 1.847 & 0.054 & 1 & 81.855 & 3.605 \\
\hline$U V R_{(N E G)}$ & 1.898 & 0.092 & 1 & $\begin{array}{c}153.35 \\
3\end{array}$ & 6.183 \\
\hline$U V R_{(N E U)}$ & 1.842 & 0.103 & 1 & $\begin{array}{c}124.47 \\
4\end{array}$ & 6.905 \\
\hline$P C I$ & 89.215 & 2.363 & 6 & 1073 & 158.877 \\
\hline
\end{tabular}

\subsection{Model specification}

In order to test the proposed hypotheses, we develop two econometrics models. In the equation (1), we tried to model the main effect of consumers' sentiments on post popularity factors (i.e. likes' count, comments' count, and shares' count):

$P P_{j i t}=\alpha+\beta_{1} P O S_{i t}+\beta_{2} N E G_{i t}+\beta_{3} N E U_{i t}+\beta_{4} P C I+\varepsilon_{i t}$ 
where $P P_{j i t}$ can be one of the $P P_{L i t}, P P_{C i t}$, or $P P_{S i t}$ that are the likes' count $(L)$, comments' count $(C)$, or shares' count $(S)$ of post $i$ at time $t$, respectively. Also, $P O S_{i t}$ is the frequency of positive comments in post $i$ at time $t, N E G_{i t}$ is the number of negative comments in post $i$ at time $t$, and finally $N E U_{i t}$ is the number of neutral comments in post $i$ at time $t$. In addition, $P C I_{i r}$ refers to the time interval between the creation time of post $i$ and time $t$. Finally, $\varepsilon_{i t}$ is the error term in the presented equation. This sort of simultaneous equations is used in prior research such as $\mathrm{Lu}$ et al. [38]; they examined the impact of online WOM on product sales using a weekly panel data.

In order to examine the effect that consumers' sentiments have on post popularity with consideration of moderating effects of consumers' up-votes, we now describe an alternative model to the model (1). But we first need to operationalize a proper measure for consumers' up-votes. In this regard, we try to measure the average count of up-votes for comments expressing each sentiment. However, there are comments with zero up-votes and the average count of up-votes for any sentiment may become zero. So, we add one to the measure to obviate this impediment [31]. Equation (2) shows the final measure of the up-votes ratio for positive comments related to post $i$ and time $t$.

$U V R_{(P O S) i t}=\left(\sum_{k=1}^{K} U V C_{(P O S) k i t} / P O S_{i t}\right)+1$

where $k$ is the comment number $(k=1,2, \ldots, \mathrm{K})$, and $U V C_{(P O S) k i t}$ is the up-vote count for comment $k$ of post $i$ at time $t$. Also, we use the same measure as equation (2) for the up-votes ratio of negative comments $\left(U V R_{(N E G) i t}\right)$ or neutral comments $\left(U V R_{(N E U) i t}\right)$. Accordingly, to investigate the effect of consumers' sentiments with consideration of the moderating variables, we use the following formulation:

$$
\begin{aligned}
P P_{j i t}= & \alpha+\beta_{1} P O S_{i t}+\beta_{2} N E G_{i t}+\beta_{3} N E U_{i t}+ \\
& \beta_{4} U V R_{(P O S) i t} \times P O S_{i t}+\beta_{5} U V R_{(N E G) i t} \times N E G_{i t}+ \\
& \beta_{6} U V R_{(N E U) i t} \times N E U_{i t}+\beta_{7} P C I+\varepsilon_{i t}
\end{aligned}
$$

\section{Empirical results}

We performed a rigorous analysis process on retrieved data to prepare the required dataset for testing the hypotheses. This dataset consists of 643 posts for each day. Due to the fact that all equations in models (1) include a same set of regressors, we employed Ordinary Least Squares (OLS) and then compared the results with outcomes of panel model estimators such as fixed-effect and random-effect estimators for each equation. We also followed the same procedure for the model (3). Fixed effect estimations outperformed OLS estimations according to the p-value $(<0.001)$. After observing the results of Hausman test and the $p$-value for each test (shown in Table 2), we decided whether to focus on fixed-effect or random-effect analysis results for each model.

Table 2. Results of the Hausman test

\begin{tabular}{|c|c|c|c|c|c|}
\hline \multicolumn{2}{|c|}{ Model } & Chi Sq. & $p$-value & $\begin{array}{c}\text { Fixed-effect } \\
\text { Analysis }\end{array}$ & $\begin{array}{c}\text { Random-effect } \\
\text { Analysis }\end{array}$ \\
\hline \multirow{3}{*}{$(1)$} & $L$ & 4.731 & 0.316 & No & Yes \\
\cline { 2 - 6 } & $C$ & 151.27 & $<0.001$ & Yes & No \\
\cline { 2 - 6 } & $S$ & 1.429 & 0.839 & No & Yes \\
\hline \multirow{3}{*}{$(3)$} & $L$ & 20.688 & 0.023 & Yes & No \\
\cline { 2 - 6 } & $C$ & 886.26 & $<0.001$ & Yes & No \\
\cline { 2 - 6 } & $S$ & 25.786 & 0.004 & Yes & No \\
\hline
\end{tabular}

Note. All numbers are rounded to three decimal digits.

We corrected our results for presence of serial correlation and heteroscedasticity by using clustered standard errors for our estimations based on Arellano's method [39]. We explain the robust results of panel analysis for the model (1) and each proxy variable of post popularity (shown in the first section of Table 3 ). All evaluations for 3 proxy variables of post popularity based on model (1) are significant as a whole ( $p$-value $<0.001)$ and their variance is well explained according to the obtained values for adjusted $R^{2}$. However, this model explains the variance of the first dependent variable (likes' count) better than the other two.

We can see that positive comments is strongly significant and related to all three proxy variables and its effect on each post popularity factor is positive. Therefore, the exposure to positive comments in postrelated comments is positively associated with the popularity of posts in social media brand communities. Thus, hypothesis $\mathrm{H} 1$ is supported according to the results related to model (1). The exposure to negative comments is positively associated with the comments' count of posts but there is no significant relationship between the exposure to negative comments and other two proxy variables of post popularity (likes' count and shares' count). Thus, we can conclude hypothesis $\mathrm{H} 2$ is not supported according to the testing by the model (1). In such situation, consumers might tent to write negative comments. Similarly, exposure to neutral comments has no significant relationship with proxy variables of post popularity. Therefore, the 
hypothesis $\mathrm{H} 3$ is not supported according to results related to the model (1).

Table 3. Empirical Results

\begin{tabular}{|c|c|c|c|c|}
\hline Model & Variables & $L$ & C & $S$ \\
\hline \multirow{6}{*}{ (1) } & $P O S$ & $\begin{array}{c}37.211 * * * \\
(7.405)\end{array}$ & $\begin{array}{c}1.418 * * * \\
(0.062)\end{array}$ & $\begin{array}{c}2.230 * * * \\
(0.401)\end{array}$ \\
\hline & $N E G$ & $\begin{array}{c}-65.675 \\
(116.819)\end{array}$ & $\begin{array}{l}1.368 * * \\
(0.474)\end{array}$ & $\begin{array}{c}1.749 \\
(8.225)\end{array}$ \\
\hline & $N E U$ & $\begin{array}{c}79.601 \\
(74.969)\end{array}$ & $\begin{array}{l}-2.384 \\
(2.180)\end{array}$ & $\begin{array}{c}2.800 \\
(3.426)\end{array}$ \\
\hline & $P C I$ & $\begin{array}{l}3.287 * * \\
(1.198)\end{array}$ & $\begin{array}{l}0.422 * \\
(0.185)\end{array}$ & $\begin{array}{c}0.251 \\
(0.324)\end{array}$ \\
\hline & Intercept & $\begin{array}{c}17374.197 * * * \\
(3574.741)\end{array}$ & - & $\begin{array}{l}2332.405^{*} \\
\text { (1149.440) }\end{array}$ \\
\hline & Adj. $R^{2}$ & 0.334 & 0.056 & 0.043 \\
\hline & & & & \\
\hline \multirow{9}{*}{ (3) } & POS & $\begin{array}{c}37.988 * * * * \\
(8.101)\end{array}$ & $\begin{array}{c}1.752 * * * \\
(0.338)\end{array}$ & $\begin{array}{c}1.774 * * * \\
(0.517)\end{array}$ \\
\hline & $N E G$ & $\begin{array}{c}-67.933 \\
(119.964)\end{array}$ & $\begin{array}{l}0.185 \\
(0.664)\end{array}$ & $\begin{array}{c}5.406 \\
(7.246)\end{array}$ \\
\hline & $N E U$ & $\begin{array}{c}71.897 \\
(75.130)\end{array}$ & $\begin{array}{l}-3.070 \\
(2.326)\end{array}$ & $\begin{array}{c}4.446 \\
(6.813)\end{array}$ \\
\hline & $\begin{array}{c}U V R_{(P O S)} \times \\
P O S\end{array}$ & $\begin{array}{l}-0.717 \\
(0.811)\end{array}$ & $\begin{array}{l}-0.198 \\
(0.219)\end{array}$ & $\begin{array}{c}0.212 \\
(0.229)\end{array}$ \\
\hline & $\begin{array}{c}U V R_{(N E G)} \times \\
N E G\end{array}$ & $\begin{array}{c}-2.847 * * * \\
(0.230)\end{array}$ & $\begin{array}{c}-0.609 * * * \\
(0.050)\end{array}$ & $\begin{array}{c}0.461 * * * * \\
(0.051)\end{array}$ \\
\hline & $\begin{array}{c}U V R_{(N E U) \times} \\
\quad N E U\end{array}$ & $\begin{array}{l}6.435 \\
(7.497)\end{array}$ & $\begin{array}{l}-0.137 \\
(1.826)\end{array}$ & $\begin{array}{c}1.981 \\
(2.025)\end{array}$ \\
\hline & $P C I$ & $\begin{array}{l}2.670 * * \\
(1.032)\end{array}$ & $\begin{array}{l}0.290 * \\
(0.124)\end{array}$ & $\begin{array}{c}0.386 \\
(0.294)\end{array}$ \\
\hline & Intercept & - & - & - \\
\hline & Adj. $R^{2}$ & 0.395 & 0.314 & 0.128 \\
\hline
\end{tabular}

Note. Statistical significance at: * 0.05 ; ** 0.01 ; *** 0.001 levels; All numbers are rounded to three decimal digits. Standard errors in parentheses.

After examining the main effects of exposure to consumers' sentiments on post popularity based on model (1), we now explain the robust results of examining those effects with consideration of the moderating role of consumers' up-votes based on model (3) which is shown in the second section of Table 3. All evaluations for 3 proxy variables of post popularity based on model (3) are significant as a whole $(p$-value $<0.001)$. We can see that the obtained adjusted $R^{2}$ for all dependent variables in the model (3) are significantly improved in comparison to the results of the model (1). It shows that model (3) is able to better explain the variances of all dependent variables. In other words, consideration of the moderation effect of consumers' up-votes in the model (3) improved the power of model in terms of explaining the variance of dependent variables in comparison to model (1).

Again, the exposure to positive comments is strongly and positively associated with all three proxy variables. Thus, hypothesis $\mathrm{H} 1$ is again supported according to the testing of the model (3). However, the exposure to negative comments has no significant relationship with all proxy variables of post popularity. Therefore, hypothesis $\mathrm{H} 2$ is not supported again according to the results related to the model (3). Also, the exposure to neutral comments has no significant relationship with all proxy variables. Similar to results of the model (1), H3 is not supported again based on the results related to the model (3).

In hypothesis $\mathrm{H} 4 \mathrm{a}$, we assumed that the up-vote ratio of positive comments amplifies the popularity of brand-related posts positively. However, our results show no significance for the moderation effect of the up-votes ratio of positive comments on post popularity. Thus, the H4a is not supported. Regarding hypothesis $\mathrm{H} 4 \mathrm{~b}$, our results show the moderation effect of the upvotes ratio of positive comments on post popularity is strongly significant. However, the direction of this moderation effect is different for each proxy variable of post popularity. Thus, we rather conclude the hypothesis H4b is partially supported. Finally, the upvotes ratio of neutral comments has no significant moderation effect on the relationship between the exposure to neutral comments and all proxy variables. Accordingly, we conclude the hypothesis $\mathrm{H} 4 \mathrm{c}$ is not supported by our results.

We can conceive from the results that when consumers express more positive sentiments in their comments, they are contributing to the popularity of the post generally. When they are impartial about a post and they express neutral sentiments about the post in their comments, it might not help the post to gain more engagement and popularity. On the other hand, when consumers express more negative sentiments in their comments in relation to a post it might be favorable for the popularity of the post because they might write negative comments and raise the comments' count. Meanwhile, if more negative comments gain more up-votes, it positively amplifies the shares' count (i.e. may stimulates consumers to share the post with their friends) and conversely inhibits likes' count and comments' count of the post (i.e. may hinder consumers to like the post or write a comment on the post).

\section{Discussions}

By concentrating on $\mathrm{C} 2 \mathrm{C}$ interactions in social media brand communities, we showed how important the consumers' sentiments embedded in post-related 
comments are and firms should pay attention to the consumers' sentiments. Our empirical analysis supports a part of findings of De Vries et al. [13]. Both studies support the fact that expression of positive sentiments through comments of a post has a positive relationship with likes' count. Also, they claimed that sharing negative comments on brand-related posts is positively associated with comments' count and our findings approve such a relationship. However, when we count for the role of up-votes, this relationship is not significant anymore.

Reacting to others' comments through up-votes is a sort of $\mathrm{C} 2 \mathrm{C}$ interaction that is investigated by a handful of sporadic studies and the existing knowledge about consequences of such online behavior is confined to a limited understanding. Thus, it needs to be investigated more. To the best of our knowledge, this is the first study examining the role of consumers' up-votes for others' comments as a moderator of the effect that consumers' sentiments may have on post popularity. Our findings on this moderation effect can be promising for developing new hypotheses and theories. However, these findings are preliminary and this moderation effect may need to be more investigated in future research in order to make a more mature body of knowledge on such online interaction.

In addition, focusing on share's count as a proxy of post popularity along with the specific effect of each sentiment feature especially neutral sentiments has been somehow overlooked in prior research as it was explained before. Therefore, this study contributes to the prior literature of social media brand community research and UGC. This research increases the existing knowledge related to the value of UGC for business. Following the findings of Tang et al. [20], our research also acknowledges that neutral comments should not be underestimated as an influential sentiment. To the best of our knowledge, this is the first study examining the effect of neutral sentiments on the popularity of the posts. Therefore, our findings are useful for directing the future research.

We showed consumers' sentiments and the way consumers express them in their comments on brandrelated posts are important to be considered by marketing practitioners. Our findings are very useful for them to improve their strategies regarding social media marketing and social media monitoring. Consumers' sentiments about brand-related posts might be derived from the content of the post. Brands should try to prepare the content of the post in a way that it gives consumers a good feeling. For instance, if the content is an advertisement, it should arouse the sense of satisfaction in consumers for the time that they spend on consuming that content. Therefore, it probably helps brands to gain more positive sentiments about their post and consequently more popularity for the post. Sometimes, consumers' sentiments might not be related to the content of brand-related posts and they might be related to their general satisfaction about brand products or services. In this case, brands should focus on the consumers' comments and find a proper way to improve their consumers' satisfaction. If firms try to remove comments that expressed negative sentiments in order to block the plausible negative effect on post popularity, it may affect consumers' general opinion about the brand and lead them to churn. One good action might be responding to consumers' comments and establishing a good relationship with their consumers.

Firms can apply our data analysis process to provide business intelligence. This process can help them to produce useful reports and insights about consumers' participation in post-related activities and their sentiments. For example, firms may find a good answer about the failure of a specific promotion by analyzing consumers' sentiments about the promotionrelated posts. Therefore, this analytical process can help firms in the efficient monitoring of their brand communities. Accordingly, this analytical process can be useful for improvement of social media management tools which are effective for acquiring attitudinal loyalty and better word-of-mouth communications [40].

This research had limitations that we would like to mention. We only considered comments written in English and all comments written in other languages is removed from the data. Also, any comment consisting of anything except text such as emoticons or hyperlinks were removed from the analysis. We focused on brands in Technology sector which are very famous and they have an enormous population of consumers. We suppose working on brands from other sectors or even infamous brands may lead to interesting results. So, this is also a direction for future research in the social media context. Investigation of other social media platforms can be a good opportunity for future research to show the generalizability of the findings.

\section{Conclusions}

Social media is supposed to be a relatively low-cost marketing channel for promoting brands. In particular, social media brand communities provide an opportunity for firms to easily disseminate brandrelated contents and garner consumers' opinions toward these contents. In this research, we mined consumers' sentiments to provide a meaningful dataset by using semi-supervised sentiment analysis, and then 
we performed a panel data analysis to examine the proposed hypotheses regarding determinants of post popularity in social media brand communities. Our findings show that exposure to positive and negative sentiments embedded in post-related comments is significantly associated with different proxies of the popularity of a brand-related post to a certain extent. However, such relationships are likely to be moderated by consumers' up-votes in the comment section of the post. This empirical study provides proper insights about the exposure to different sentiments through consumers' comments, the role of consumers' upvotes, and popularity of brand-related contents in brand communities of social media. Our study contributes to existing literature associated with the value of UGC for business and particularly marketing. This study has an important managerial implication for firms and their social media marketers by providing a clear understanding of consumers' sentiments and up-votes. They can use our findings to improve their strategies for social media marketing, and monitoring of their social media brand communities.

\section{Acknowledgment}

This work was supported by grants from the Research Grants Council of the Hong Kong Special Administrative Region, China (Project: CityU 11502115), the Shenzhen Municipal Science and Technology R\&D Funding - Basic Research Program (Projects: JCYJ20140419115614350 and JCYJ20160229165300897), and City University of Hong Kong (Project No. 7200355).

\section{References}

[1] M. R. Habibi, M. Laroche, and M.-O. Richard, "Brand communities based in social media: How unique are they? Evidence from two exemplary brand communities," International Journal of Information Management, vol. 34, no. 2, pp. 123-132, 2014.

[2] M. Laroche, M. R. Habibi, and M.-O. Richard, "To be or not to be in social media: How brand loyalty is affected by social media?," International Journal of Information Management, vol. 33, no. 1, pp. 76-82, 2013.

[3] K. Heinonen, "Consumer activity in social media: Managerial approaches to consumers' social media behavior," Journal of Consumer Behaviour, vol. 10, no. 6, pp. 356-364, 2011.

[4] K. Xie and Y.-J. Lee, "Social Media and Brand Purchase: Quantifying the Effects of Exposures to Earned and Owned Social Media Activities in a TwoStage Decision Making Model," Journal of Management Information Systems, vol. 32, no. 2, pp. 204-238, 2015.
[5] H. Khobzi and B. Teimourpour, "How significant are users' opinions in social media?," International Journal of Accounting \& Information Management, vol. 22, no. 4, pp. 254-272, 2014.

[6] R. Rishika, A. Kumar, R. Janakiraman, and R. Bezawada, "The effect of customers' social media participation on customer visit frequency and profitability: an empirical investigation," Information systems research, vol. 24, no. 1, pp. 108-127, 2013.

[7] K.-Y. Goh, C.-S. Heng, and Z. Lin, "Social media brand community and consumer behavior: Quantifying the relative impact of user-and marketergenerated content," Information Systems Research, vol. 24, no. 1, pp. 88-107, 2013.

[8] J. Q. Dong and W. Wu, "Business value of social media technologies: Evidence from online user innovation communities," The Journal of Strategic Information Systems, vol. 24, no. 2, pp. 113-127, 2015.

[9] H. S. Manaman, S. Jamali, and A. AleAhmad, "Online reputation measurement of companies based on usergenerated content in online social networks," Computers in Human Behavior, vol. 54, pp. 94-100, 2016.

[10] B. Libai, R. Bolton, M. S. Bügel, K. De Ruyter, O. Götz, H. Risselada, and A. T. Stephen, "Customer-tocustomer interactions: broadening the scope of word of mouth research," Journal of Service Research, vol. 13, no. 3, pp. 267-282, 2010.

[11] C. M. Cheung, B. S. Xiao, and I. L. Liu, "Do actions speak louder than voices? The signaling role of social information cues in influencing consumer purchase decisions," Decision Support Systems, vol. 65, pp. 5058, 2014.

[12] H. Chen, R. H. Chiang, and V. C. Storey, "Business Intelligence and Analytics: From Big Data to Big Impact.," MIS quarterly, vol. 36, no. 4, pp. 11651188, 2012.

[13] L. De Vries, S. Gensler, and P. S. Leeflang, "Popularity of brand posts on brand fan pages: an investigation of the effects of social media marketing," Journal of Interactive Marketing, vol. 26, no. 2, pp. 83-91, 2012.

[14] F. Sabate, J. Berbegal-Mirabent, A. Cañabate, and P. R. Lebherz, "Factors influencing popularity of branded content in Facebook fan pages," European Management Journal, vol. 32, no. 6, pp. 1001-1011, 2014.

[15] A. H. Zadeh and R. Sharda, "Modeling brand post popularity dynamics in online social networks," Decision Support Systems, vol. 65, pp. 59-68, 2014.

[16] Y. Ding, C. W. Phang, X. Lu, C.-H. Tan, and J. Sutanto, "The role of marketer-and user-generated content in sustaining the growth of a social media brand community," in System Sciences (HICSS), 2014 47th Hawaii International Conference on, 2014, pp. 1785-1792.

[17] M. Scholz, V. Dorner, A. Landherr, and F. Probst, "Awareness, interest, and final decision: The effects of user-and marketer-generated content on consumers' 
purchase decisions," in ICIS 2013 Proceedings, Milan, Italy, 2013, p. Paper 23.

[18] Y.-Y. Wang, A. Susarla, and V. Sambamurthy, "The Untold Story of Social Media on Offline Sales: The Impact of Facebook in the US Automobile Industry," in ICIS 2015 Proceedings, Fort Worth, 2015, p. Paper 3.

[19] Z. Lin and K. Y. Goh, "Measuring the business value of online social media content for marketers," in ICIS 2011 Proceedings, 2011, p. Paper 16.

[20] T. Tang, E. Fang, and F. Wang, "Is neutral really neutral? The effects of neutral user-generated content on product sales," Journal of Marketing, vol. 78, no. 4, pp. 41-58, 2014.

[21] J. Wu, L. Huang, J. L. Zhao, and Z. Hua, "The deeper, the better? Effect of online brand community activity on customer purchase frequency," Information \& Management, vol. 52, no. 7, pp. 813-823, 2015.

[22] H.-Y. Tsao, P. Berthon, L. F. Pitt, and M. Parent, "Brand signal quality of products in an asymmetric online information environment: An experimental study," Journal of Consumer Behaviour, vol. 10, no. 4, pp. 169-178, 2011.

[23] T. Mavlanova, R. Benbunan-Fich, and M. Koufaris, "Signaling theory and information asymmetry in online commerce," Information \& Management, vol. 49, no. 5, pp. 240-247, 2012.

[24] M. Thongmak, "Engaging Facebook Users in Brand Pages: Different Posts of Marketing-Mix Information," in Business Information Systems, 2015, pp. 299-308.

[25] T.-P. Liang, X. Li, C.-T. Yang, and M. Wang, "What in consumer reviews affects the sales of mobile apps: A multifacet sentiment analysis approach," International Journal of Electronic Commerce, vol. 20, no. 2, pp. 236-260, 2015.

[26] H. Rui, Y. Liu, and A. Whinston, "Whose and what chatter matters? The effect of tweets on movie sales," Decision Support Systems, vol. 55, no. 4, pp. 863-870, 2013.

[27] G. P. Sonnier, L. McAlister, and O. J. Rutz, "A dynamic model of the effect of online communications on firm sales," Marketing Science, vol. 30, no. 4, pp. 702-716, 2011.

[28] L. Muchnik, S. Aral, and S. J. Taylor, "Social influence bias: A randomized experiment," Science, vol. 341, no. 6146, pp. 647-651, 2013.

[29] M. Priestley and A. Mesoudi, "Do Online Voting Patterns Reflect Evolved Features of Human Cognition? An Exploratory Empirical Investigation," PloS one, vol. 10, no. 6, p. e0129703, 2015.
[30] B. Stephens, W. Chen, and J. S. Butler, "Bubbling Up the Good Ideas: A Two-Mode Network Analysis of an Intra-Organizational Idea Challenge," Journal of Computer-Mediated Communication, vol. 21, no. 3, pp. 210-229, 2016.

[31] P. T. Ngoc and M. Yoo, "The lexicon-based sentiment analysis for fan page ranking in Facebook," in Information Networking (ICOIN), 2014 International Conference on, 2014, pp. 444-448.

[32] B.-C. Chen, J. Guo, B. Tseng, and J. Yang, "User reputation in a comment rating environment," in Proceedings of the 17th ACM SIGKDD international conference on Knowledge discovery and data mining, 2011, pp. 159-167.

[33] V. Noguti, N. Lee, and Y. Dwivedi, "Post language and user engagement in online content communities," European Journal of Marketing, vol. 50, no. 5/6, p. , 2016.

[34] A. Majchrzak, S. Faraj, G. C. Kane, and B. Azad, "The contradictory influence of social media affordances on online communal knowledge sharing," Journal of Computer-Mediated Communication, vol. 19, no. 1, pp. 38-55, 2013.

[35] A. Rechavi and S. Rafaeli, "Knowledge and social networks in Yahoo! Answers," in System Science (HICSS), 2012 45th Hawaii International Conference on, 2012, pp. 781-789.

[36] J. Wiebe and R. Mihalcea, "Word sense and subjectivity," in Proceedings of the 21st International Conference on Computational Linguistics and the 44th annual meeting of the Association for Computational Linguistics, 2006, pp. 1065-1072.

[37] Y. Bengio, O. Delalleau, and N. Le Roux, "Label propagation and quadratic criterion," in Semisupervised learning, England: The MIT Press, 2006, pp. 193-216.

[38] X. Lu, S. Ba, L. Huang, and Y. Feng, "Promotional marketing or word-of-mouth? Evidence from online restaurant reviews," Information Systems Research, vol. 24, no. 3, pp. 596-612, 2013.

[39] M. Arellano, "PRACTITIONERS'CORNER: Computing Robust Standard Errors for Within-groups Estimators," Oxford bulletin of Economics and Statistics, vol. 49, no. 4, pp. 431-434, 1987.

[40] M. Risius and R. Beck, "Effectiveness of corporate social media activities in increasing relational outcomes," Information \& Management, vol. 52, no. 7, pp. 824-839, 2015. 